\title{
Supporting Information: Proximate quantum spin liquid on designer lattice
}

\author{
Xiaoran Liu, ${ }^{*, \dagger}$ Sobhit Singh, ${ }^{\dagger}$ Victor Drouin-Touchette,${ }^{\dagger}$ Tomoya Asaba, ${ }^{\dagger}$ \\ Jess Brewer, ${ }^{\top, \S}$ Qinghua Zhang,\| Yanwei Cao, ${ }^{\perp}$ Banabir Pal, ${ }^{\dagger, \#}$ Srimanta \\ Middey, ${ }^{@}$ P. S. Anil Kumar, ${ }^{\circledR}$ Mikhail Kareev,${ }^{\dagger}$ Lin Gu,, D. D. Sarma, ${ }^{\#}$ \\ Padraic Shafer, ${ }^{\triangle}$ Elke Arenholz, ${ }^{\triangle}$ John W. Freeland, ${ }^{\nabla}$ Lu Li, ${ }^{\ddagger}$ David \\ Vanderbilt, $^{\dagger}$ and Jak Chakhalian ${ }^{\dagger}$
}

$\dagger$ †epartment of Physics and Astronomy, Rutgers University, Piscataway, New Jersey 08854, USA $\ddagger$ Department of Physics, University of Michigan, Ann Arbor, Michigan 48109, USA

ФTRIUMF, 4004 Wesbrook Mall, Vancouver, British Columbia, Canada V6T 2A3

$\S$ Department of Physics and Astronomy, University of British Columbia, Vancouver, British Columbia, Canada V6T 1Z1

||Beijing National Laboratory for Condensed-Matter Physics and Institute of Physics, Chinese Academy of Sciences, Beijing 100190, P. R. China

$\perp$ Ningbo Institute of Materials Technology and Engineering, Chinese Academy of Sciences, Ningbo, Zhejiang 315201, China

\#Solid State and Structural Chemistry Unit, Indian Institute of Science, Bengaluru 560012, India @Department of Physics, Indian Institute of Science, Bengaluru 560012, India $\triangle$ Advanced Light Source, Lawrence Berkley National Laboratory, Berkeley, California 94720 , USA

$\nabla$ Advanced Photon Source, Argonne National Laboratory, Argonne, Illinois 60439, USA

E-mail: xiaoran.liu@rutgers.edu 


\section{Sample Fabrications}

The $\left[n \text { QL } \mathrm{CoCr}_{2} \mathrm{O}_{4} / 1.3 \mathrm{~nm} \mathrm{Al}_{2} \mathrm{O}_{3}\right]_{4}(n=1,2,4 ; 1 \mathrm{QL} \approx 4.8 \AA)$ superlattices were grown on 5 $\times 5 \mathrm{~mm}^{2} \mathrm{Al}_{2} \mathrm{O}_{3}$ (0001) substrates by pulsed laser deposition. Stoichiometric $\mathrm{CoCr}_{2} \mathrm{O}_{4}$ and $\mathrm{Al}_{2} \mathrm{O}_{3}$ targets were ablated using a $\mathrm{KrF}$ excimer laser $\left(\lambda=248 \mathrm{~nm}\right.$, energy density $\left.\sim 2 \mathrm{~J} / \mathrm{cm}^{2}\right)$ with a repetition rate of $4 \mathrm{~Hz}$ and $2 \mathrm{~Hz}$, respectively. The depositions were carried out at a substrate temperature of $700{ }^{\circ} \mathrm{C}$, under oxygen partial pressure of 5 mTorr. The films were post-annealed at the growth condition for 15 minutes and then cooled down to room temperature at $10^{\circ} \mathrm{C} / \mathrm{min}$. The deposition process was monitored by in-situ high pressure reflection-high-energy-electron-diffraction (RHEED). Furthermore, quality of the superlattices has been verified by using a variety of ex-situ characterization methods including synchrotron-based x-ray diffraction, x-ray reflectivity, x-ray photoemission spectroscopy and atomic force microscopy, as published elsewhere. ${ }^{1,2}$

\section{Magnetization}

In order to probe the critical temperature $\mathrm{T}_{c}$ of the magnetic phase transition, we have also investigated the temperature dependence of the $d c$ magnetization on each sample (see Supplemental Fig. S1). The measurements were performed using the Quantum Design MPMS SQUID magnetometer. As shown on the graph, the para- to ferri- magnetic transition still takes place at $\mathrm{T}_{C} \sim 58$ $\mathrm{K}$ on $n=4$ and $\sim 40 \mathrm{~K}$ on $n=2$, respectively, whereas no clear signature of any transition was detected on the $n=1$ sample down to $2 \mathrm{~K}$.

\section{Resonant X-ray Absorption Spectra}

The local chemical states and environments of both Co and $\mathrm{Cr}$ cations of each sample were explored by measuring the resonant X-ray absorption Spectra (XAS). The experiments were performed at the beamline 4-ID-C of the Advanced Photon Source in Argonne National Laboratory. As shown in Supplemental Fig. S2, no signs of cation distribution disorder or variation of ion 


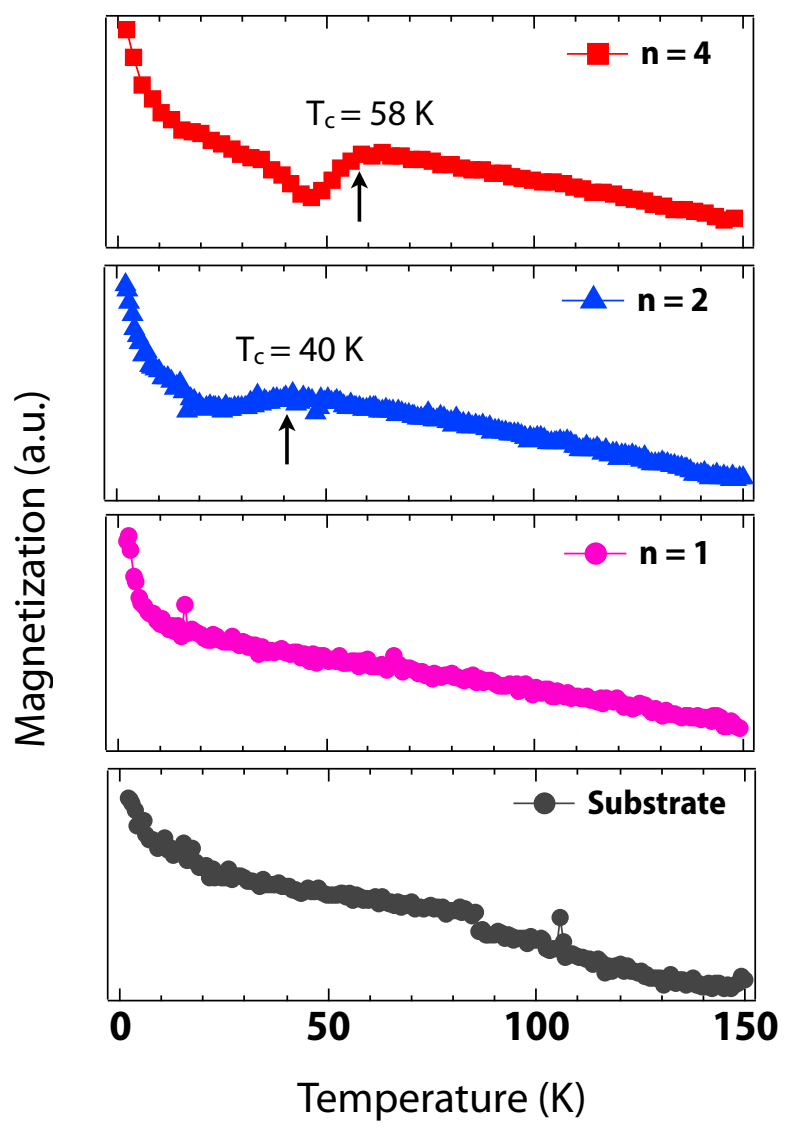

Figure S1: Temperature dependence of magnetization of $\left[n \mathrm{QL} \mathrm{CoCr}_{2} \mathrm{O}_{4} / 1.3 \mathrm{~nm} \mathrm{Al} \mathrm{O}_{3}\right]_{4}(n=1$, $2,4)$, together with a bare $\mathrm{Al}_{2} \mathrm{O}_{3}$ substrate for comparison.

valency were observed for all samples as deduced from the absorption line-shapes as well as the absorption peak positions at each $L_{2,3}$ absorption edge, which are practically identical to the bulk references (i.e., all of the Co ions are tetrahedrally coordinated with +2 valency, while all $\mathrm{Cr}$ ions are octahedrally coordinated with +3 valency). These measurements corroborate the formation of the expected ionic layers as described in the main text.

\section{X-ray Magnetic Circular Dichroism}

X-ray magnetic circular dichroism (XMCD) spectra under 3 T external field were performed at the beamline 4-ID-C of the Advanced Photon Source in Argonne National Laboratory. The $L_{2,3}$-edge X-ray absorption spectra of both Co and $\mathrm{Cr}$ were scanned with left- and right- polarized X-rays at 

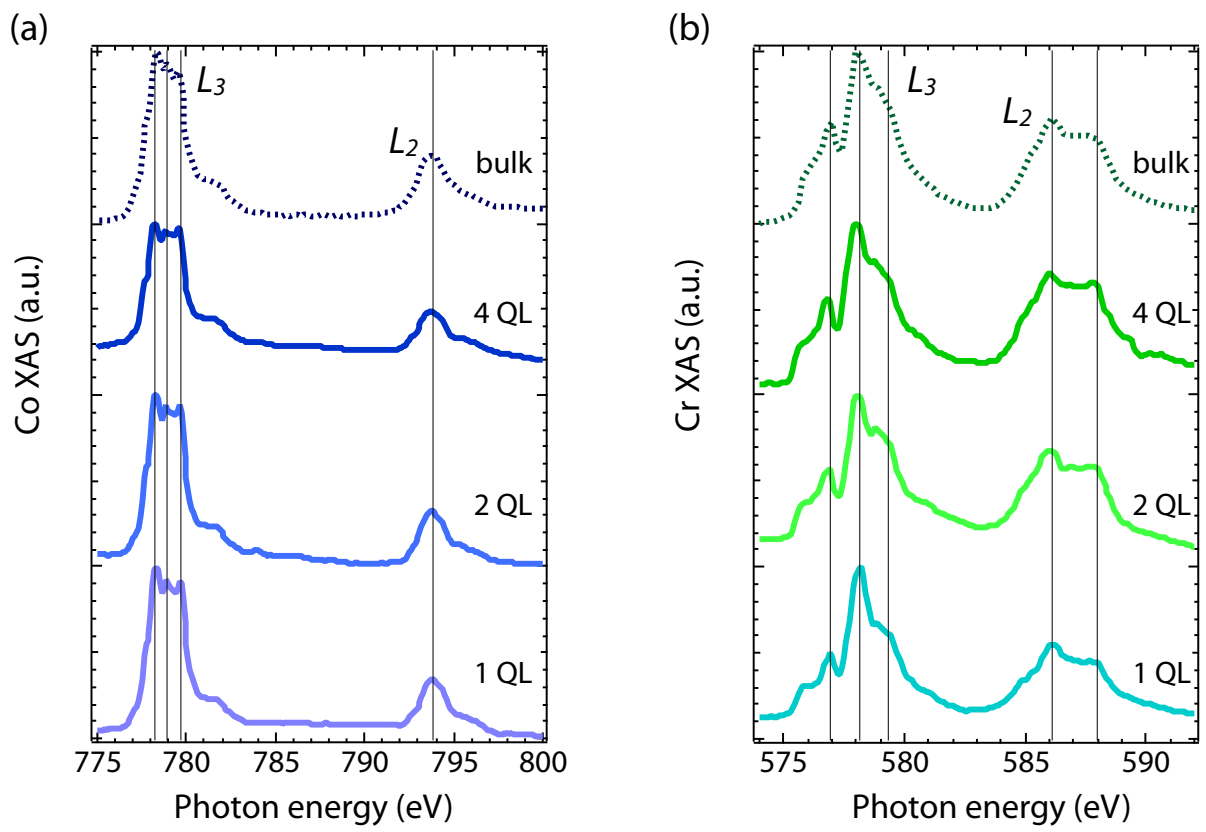

Figure S2: (a) Co and (b) $\mathrm{Cr} \mathrm{L}_{2,3}$-edges x-ray absorption spectra of the superlattices with $n=4,2$, and 1. Data were collected in the total electron yield (TEY) mode at $10 \mathrm{~K}$. The spectra taken from measurements on bulk $\mathrm{CoCr}_{2} \mathrm{O}_{4}$ powders ${ }^{3}$ are plotted in dashed lines as references.

grazing incidence at $10 \mathrm{~K}$. To exclude any artifact, all the measurements were conducted in both positive and negative external field. Data were recorded simultaneously with total electron yield (TEY), fluorescence yield (FY), and reflectivity detection modes.

The XMCD hysteresis loops were measured at the beamline 4.0.2 of the Advanced Light Source in Lawrence Berkeley National Laboratory. Samples were cooled with zero field and maintained at $15 \mathrm{~K}$. The circularly polarized soft X-rays were incident with an angle of $35^{\circ}$ relative to the sample surface. The luminescence detection mode was used to record the data.

\section{Torque Magnetometry Measurements}

Torque magnetometry measurements were performed with home-built cantilever setup by attaching samples to a thin beryllium copper cantilever. External magnetic field was applied, and the sample rotation by torque was measured by tracking the capacitance change between the metallic cantilever and a fixed gold film underneath using an AH2700A capacitance bridge with a 14 
(a)

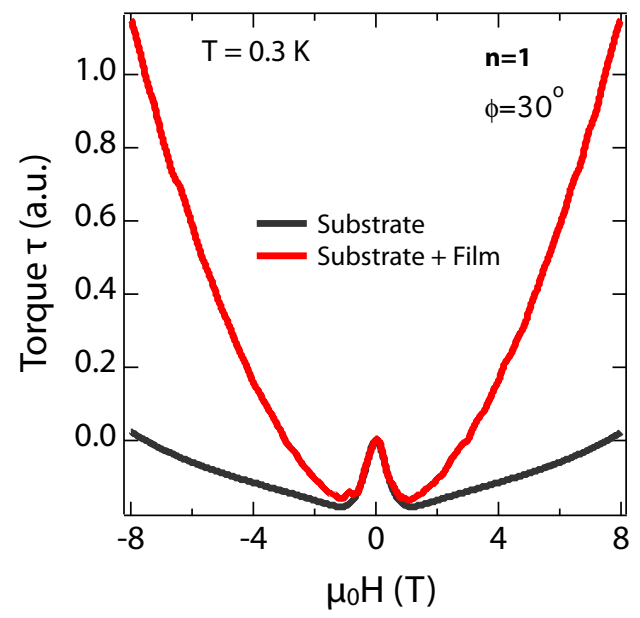

(b)

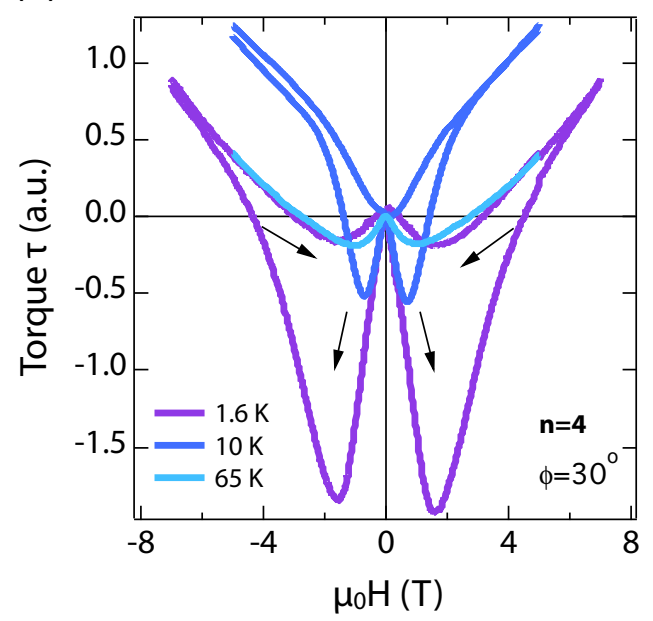

Figure S3: (a) Torque magnetometry curves of both the bare $\mathrm{Al}_{2} \mathrm{O}_{3}$ substrate (black) and the $n=$ 1 sample (red) at $0.3 \mathrm{~K}$. (b) Torque magnetometry curves of the $n=4$ sample $\left(\mathrm{T}_{C} \sim 58 \mathrm{~K}\right)$ at 1.6 $\mathrm{K}, 10 \mathrm{~K}$, and $65 \mathrm{~K}$.

$\mathrm{kHz}$ driving frequency. To calibrate the spring constant of the cantilever, we tracked the angular dependence of capacitance caused by the sample weight at zero magnetic field.

As discussed in the main text, the torque signal from the pure film is calculated by subtracting the substrate contribution at each temperature below 30 K. Here in Supplemental Fig. S3(a), we show one set of the representative measurement performed at $0.3 \mathrm{~K}$. The geometry of the setup is identical to that described in the main text. Note, the substrate was treated using the same thermal process as growing the film. As clearly seen, there is no observable magnetic hysteresis and the "bump" near zero field regime is from the substrate.

Compared to the behavior of $n=1$, the torque data of $n=4$ reveal remarkable distinction, as displayed in Supplemental Fig. S3(b). Above $\mathrm{T}_{C} \sim 58 \mathrm{~K}$, no hysteresis loop is seen, whereas below $\mathrm{T}_{C}$, distinct loops are observed. These measurements are consistent with results from SQUID and XMCD, providing another solid evidence on the establishment of long-range ferrimagnetic ordering in $n=4$, which in addition highlights the particularity of $n=1$. 


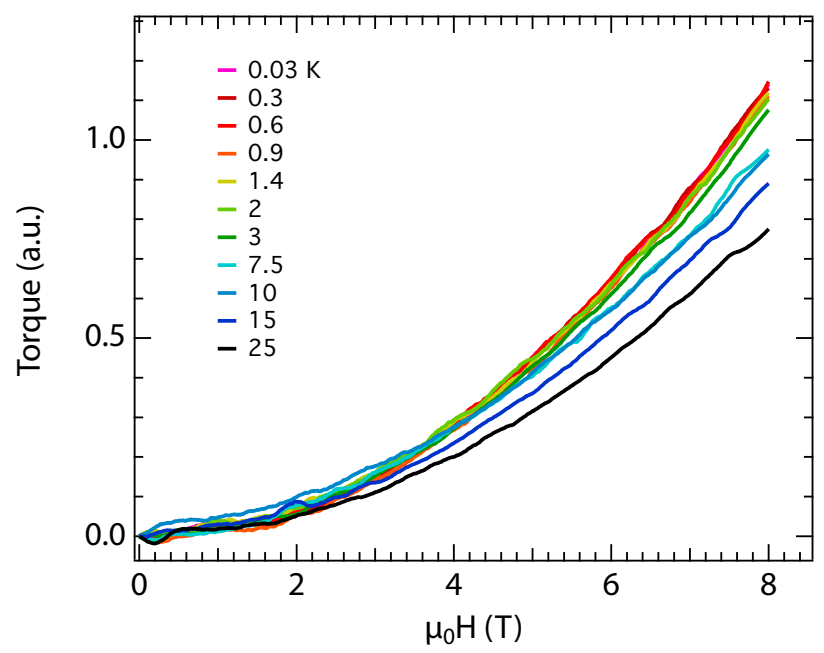

Figure S4: Torque magnetometry curves as a function of field of the $1 \mathrm{QL} \mathrm{CoCr}_{2} \mathrm{O}_{4}$ system between $30-0.03 \mathrm{~K}$.

\section{Muon Spin Relaxation Spectra}

Positive muon spin relaxation $\left(\mu^{+} \mathrm{SR}\right)$ experiments were carried out in both zero magnetic field (ZF) and a $30 \mathrm{G}$ weak transverse field (wTF) on the LEM (Low-Energy Muon) spectrometer at PSI (the Paul Scherrer Institut) in Switzerland. Five identical pieces of the $n=1$ samples on $\mathrm{Al}_{2} \mathrm{O}_{3}$ substrate were mounted on a nickel backing in order to completely depolarize any muons missing the samples. Thanks to the momentum dispersion introduced by passage through the thin carbon foil that produces free electrons to trigger the incoming muon counter, the time of flight from that foil to the sample is broadened, causing a reduced time resolution and a distorted decay spectrum over the first $\sim 0.2 \mu \mathrm{s}$. Oscillations faster than about $10 \mathrm{MHz}$ (corresponding to local fields of over $\sim 70 \mathrm{mT}$ ) cannot be resolved. The measurements were performed at a set of temperatures in the range of 4 - $100 \mathrm{~K}$, exhibiting similar behaviors. In particular, no oscillatory signal is observed in any of the $\mathrm{ZF}-\mu^{+} \mathrm{SR}$ spectra down to and including $4 \mathrm{~K}$, while a slowly relaxing signal precessing at the muon Larmor frequency is clearly observed in all of the wTF- $\mu^{+}$SR spectra. The spectra of both ZF- $\mu^{+}$SR and wTF- $\mu^{+}$SR at each temperature with fitting are shown in Supplemental Fig. S5.

The wTF- and ZF- $\mu^{+}$SR time spectra were fit simultaneously with a common slowly-relaxing 

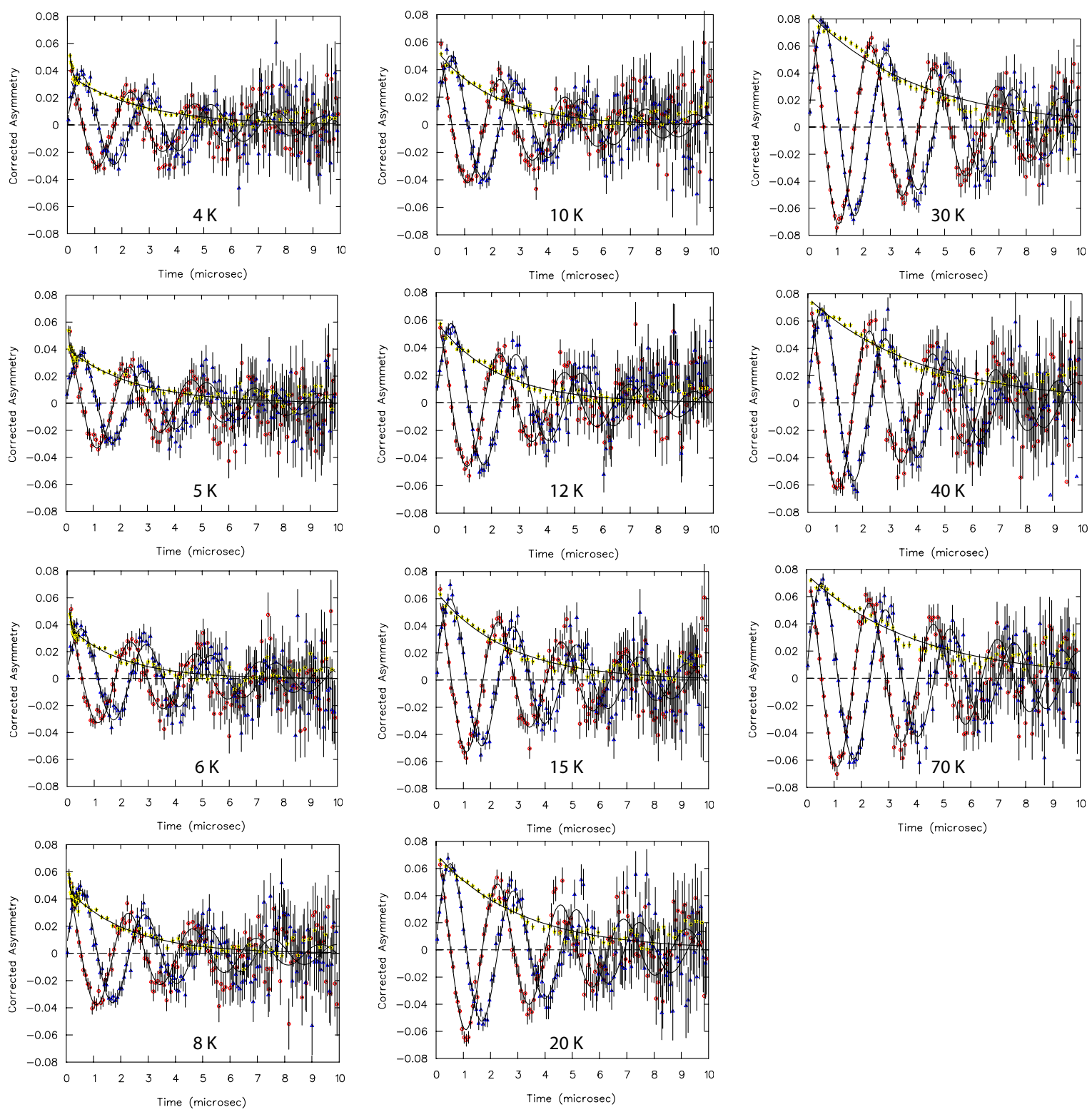

Figure S5: Spectra of the time evolution of muon-spin polarization in both zero-field (ZF, yellow stars) and $30 \mathrm{G}$ transverse field (TF, red circles [real part] and blue triangles [imaginary part]) at 11 temperatures within $4-70 \mathrm{~K}$. The spectra measured at these temperatures were fit using the equations as discussed in the text. 
asymmetry $A_{\text {slow }}$ but all other parameters independent. At temperatures below $10 \mathrm{~K}$, an additional fast-relaxing component was apparent in the ZF spectra, but not in the wTF spectra. We believe this to be due to the systematic leakage of uncorrelated beam particles, and so the fast-relaxing asymmetry reveals no physical information about the sample. Simple exponential relaxation was assumed throughout, as fits with more exotic relaxation shapes were not appreciably better. Thus the $\mathrm{ZF}-\mu^{+}$SR data below $10 \mathrm{~K}$ were fit with the sum of a fast exponential relaxation and a slow exponential relaxation:

$$
A_{0} P_{\mathrm{ZF}}(t)=A_{\text {fast }} e^{-\lambda_{\text {fast }} t}+A_{\text {slow }} e^{-\lambda_{\mathrm{ZF}} t}
$$

in which $A_{0}$ is the overall initial asymmetry and $P_{\mathrm{ZF}}(t)$ is the muon spin-polarization relaxation function. The asymmetries $\left(A_{\text {fast }}, A_{\text {slow }}\right)$ and the slow relaxation rate $\left(\lambda_{\mathrm{ZF}}\right)$ are the important fitting parameters. The slow terms $\left(A_{\text {slow }}\right.$ and $\left.\lambda_{\mathrm{ZF}}\right)$ are related to the actual muon spin relaxation inside the sample, which are the subjects of interest. At the same time, the wTF- $\mu^{+}$SR data was fit to a single exponentially relaxing precession at the frequency corresponding to the applied field:

$$
A_{0} P_{\mathrm{wTF}}(t)=A_{\mathrm{slow}} e^{-\lambda_{\mathrm{TF}} t} \cos \left(\omega_{\mu} t+\phi\right)
$$

in which $\omega_{\mu}$ is the muon Larmor frequency $\left(\omega_{\mu}=\gamma_{\mu} H, \gamma_{\mu}=2 \pi \times 135.54 \mathrm{MHz} / \mathrm{T}\right)$, and $\phi$ is the initial phase of precession. These, the asymmetry $A_{\text {slow }}$ and the relaxation rate $\lambda_{\text {TF }}$ are the important fitting parameters.

The temperature dependence of the common slow asymmetry $\left(A_{\text {slow }}\right)$ and both relaxation rates ( $\lambda_{\mathrm{ZF}}$ and $\lambda_{\mathrm{TF}}$ ) are plotted in Fig. S6, showing a consistent trend. While the asymmetry decreases at low temperature, both relaxation rates first increase, reaching a "peak" at around 9-10 K, followed by a reduction again at lower temperatures. The ZF relaxation rate is consistently about twice as fast as the TF relaxation rate at the same temperature.

In addition to runs with a muon energy of $2 \mathrm{keV}$ (optimizing the muon range in the middle of the film) a separate set of runs at $10 \mathrm{keV}$ were taken to probe the $\mathrm{Al}_{2} \mathrm{O}_{3}$ substrate. The signal amplitude in the latter runs was found to increase at low temperature, unlike that in the superlattice, 

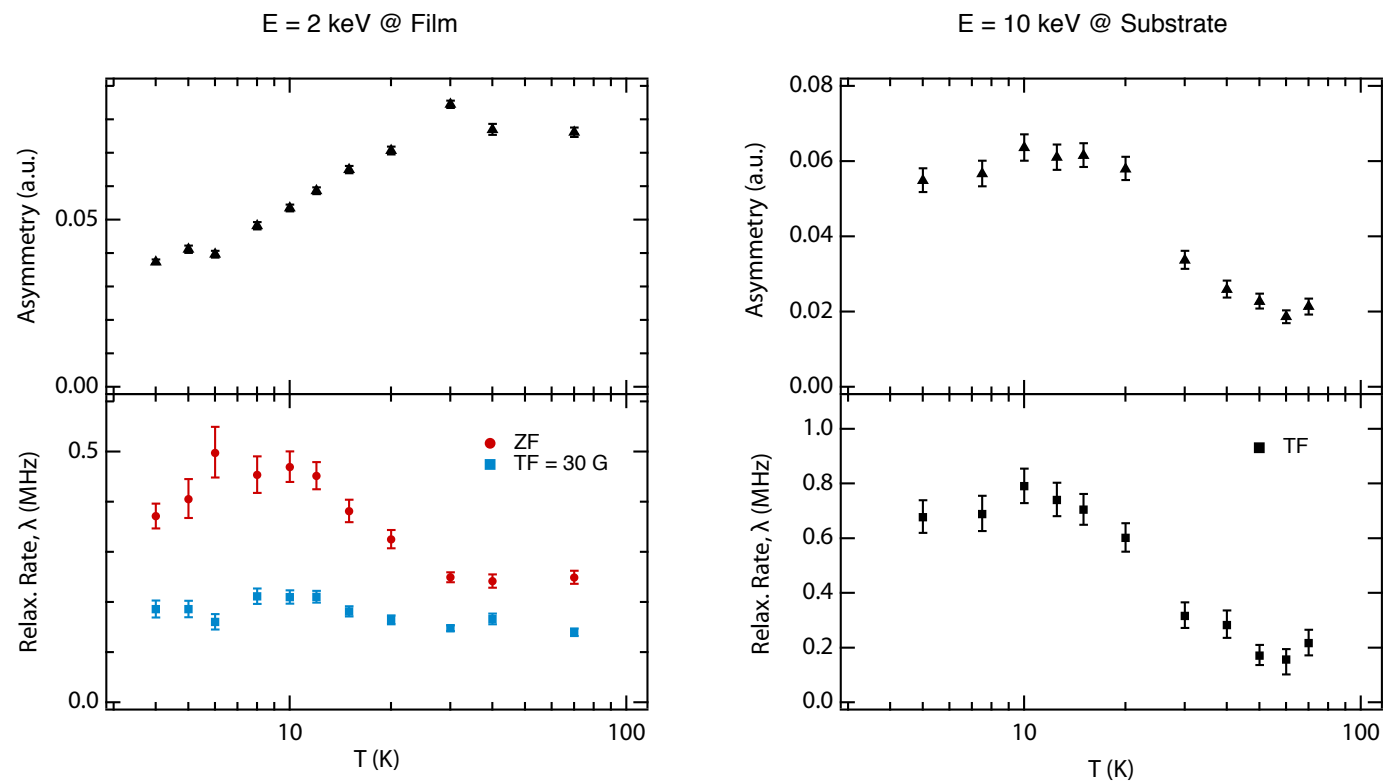

Figure S6: Asymmetry and relaxation rate of both film (left) and substrate (right) as a function of temperature.

which decreases. Moreover, the relaxation rate in $\mathrm{Al}_{2} \mathrm{O}_{3}$ (which also increases with decreasing $T$ ) is more than three times as fast as in the superlattice at base temperature. The fraction of muons in the superlattice that are not immediately depolarized at base temperature therefore cannot be just those stopping in the $\mathrm{Al}_{2} \mathrm{O}_{3}$ layers, unless such thin sheets of $\mathrm{Al}_{2} \mathrm{O}_{3}$ behave completely differently from bulk $\mathrm{Al}_{2} \mathrm{O}_{3}$.

\section{Density-Functional Theory Calculations}

Density-functional theory (DFT) based first-principles calculations were performed using the Vienna $a b$ initio simulation package (VASP) ${ }^{4,5}$ within the projected-augmented wave (PAW) framework. ${ }^{6,7}$ Nine valence electrons of $\mathrm{Co}\left(3 d^{7} 4 s^{2}\right)$, twelve valence electrons of $\mathrm{Cr}\left(3 p^{6} 3 d^{5} 4 s^{1}\right)$, and six valence electrons of $\mathrm{O}\left(2 s^{2} 2 p^{4}\right)$ were considered in the PAW pseudo-potentials. The generalized gradient approximation (GGA) as parametrized by Perdew-Burke-Ernzerhof (PBE) ${ }^{8}$ was used for the exchange-correlation functional. We use Dudarev's approach ${ }^{9}$ to include the effects arising due to the strongly correlated nature of $3 d$ electrons at the mean-field level. The PBE $+U$ scheme was 
employed, where $U_{C o}=3.3 \mathrm{eV}$ and $U_{C r}=3.7 \mathrm{eV}$ were used for the on-site Hubbard interaction terms for the $3 d$ electrons of $\mathrm{Co}$ and $\mathrm{Cr}$. These values of Hubbard parameter are reported to correctly describe the structural phase transitions and the magnetic ground state of bulk $\mathrm{CoCr}_{2} \mathrm{O}_{4} \cdot{ }^{10-13}$ We used an $8 \times 8 \times 8$ Monkhorst-Pack type $k$-mesh to sample the Brillouin zone. $600 \mathrm{eV}$ was set as the the cutoff for the kinetic energy of plane waves. $10^{-3} \mathrm{eV} / \AA$, and $10^{-8} \mathrm{eV}$ was used as the convergence criteria for residual forces and total energy in the self-consistent DFT calculations, respectively. All DFT calculations were performed in collinear magnetic setting.

The structural primitive cell of $\mathrm{CoCr}_{2} \mathrm{O}_{4}$ contains two formula units of $\mathrm{CoCr}_{2} \mathrm{O}_{4}$ with a total of six magnetic cations (2 Co and $4 \mathrm{Cr}$ ), as shown in Fig. S7(a). The optimized lattice parameters of bulk $\mathrm{CoCr}_{2} \mathrm{O}_{4}$ are $a=b=c=8.34 \AA$, with $x=0.26$ as an internal structural parameter for oxygen atoms in the cubic spinel structure (space group $F d \overline{3} m$ ). The $\mathrm{Co}-\mathrm{O}, \mathrm{Cr}-\mathrm{O}$, and $\mathrm{Cr}-\mathrm{Cr}$ bond lengths in the DFT optimized cell are $\sim 2.00, \sim 2.03$, and $\sim 3.00 \AA$, respectively. Our DFT calculations, using the aforementioned parameters, predict a Neél-type magnetic configuration as the magnetic ground state of bulk $\mathrm{CoCr}_{2} \mathrm{O}_{4}$ in which all $\mathrm{Co}$ atoms are in a spin-up configuration ( $\left.\uparrow \uparrow\right)$ and all $\mathrm{Cr}$ atoms are in a spin-down configuration $(\downarrow \downarrow \downarrow \downarrow)$. The total magnetic moment of Co and $\mathrm{Cr}$ atoms remained around $2.7 \mu_{B}$ and $3.0 \mu_{B}$, respectively, in our DFT calculations.

To simulate a slab geometry corresponding to $n=1$ layer, we created an epitaxial heterostructures containing 1 unit cell of $\mathrm{CoCr}_{2} \mathrm{O}_{4}$ sandwiched between a spacer layer of nonmagnetic and insulating $\mathrm{MgAl}_{2} \mathrm{O}_{4}$, as shown in Fig. S7(b). The details of $n=2$ heterostructure are given in Ref. ${ }^{16}$ Note, since the sole purpose here is to use a nonmagnetic material which prevents the magnetic interactions between the periodical images of $\mathrm{CoCr}_{2} \mathrm{O}_{4}$ film, we have chosen $\mathrm{MgAl}_{2} \mathrm{O}_{4}$ as the spacer material (in place of $\mathrm{Al}_{2} \mathrm{O}_{3}$ ) for purposes of computational simplicity and efficiency. We ensured that the thickness of the $\mathrm{MgAl}_{2} \mathrm{O}_{4}$ spacer layer is sufficient to prevent the magnetic interactions between the adjacent $\mathrm{CoCr}_{2} \mathrm{O}_{4}$ films. The $\mathrm{CoCr}_{2} \mathrm{O}_{4} / \mathrm{MgAl}_{2} \mathrm{O}_{4}$ heterostructure was optimized using the same convergence criteria as used for the bulk $\mathrm{CoCr}_{2} \mathrm{O}_{4}$, however, a relatively smaller Monkhorst-Pack type $k$-mesh of size $8 \times 8 \times 6$ was used to sample the Brillouin zone. $\mathrm{Mg}\left(3 s^{2}\right)$ and $\mathrm{Al}\left(3 s^{2} 3 p^{1}\right)$ PAW pseudo-potentials were used. This slab heterostructure geometry 
is similar to the one used for our experiments.

(a)

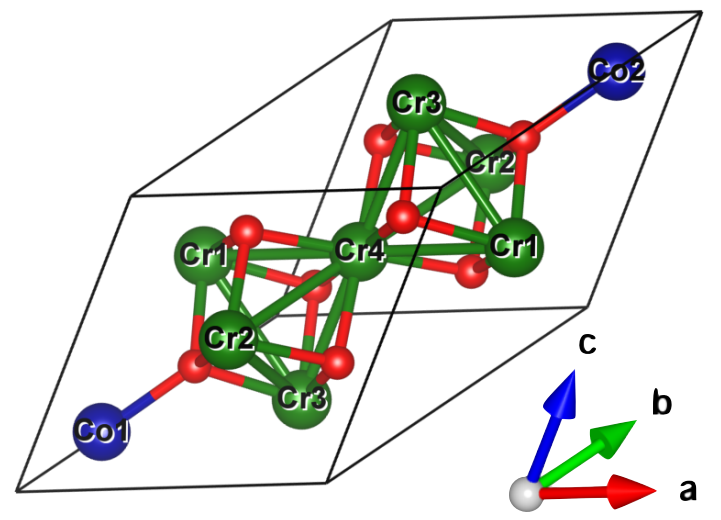

(b)

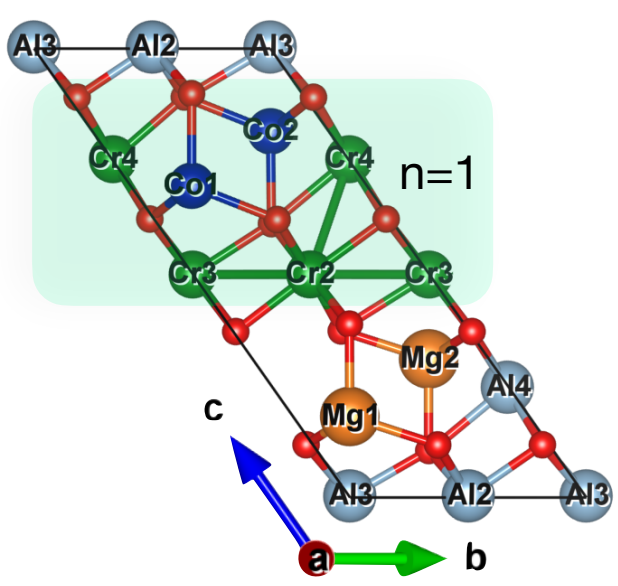

Figure S7: (a) The primitive cell of bulk $\mathrm{CoCr}_{2} \mathrm{O}_{4}$. Red, blue, and green colors depict oxygen, cobalt and chromium atoms, respectively. (b) Side view of $n=1$ epitaxial $\mathrm{CoCr}_{2} \mathrm{O}_{4} / \mathrm{MgAl}_{2} \mathrm{O}_{4}$ heterostructure.

\section{Magnetic Exchange Interaction Parameters}

In order to determine the magnetic exchange constants $\left(J_{i j}\right)$ between the nearest $\left(J_{i j}^{1}\right)$, and the second-near $\left(J_{i j}^{2}\right)$ neighbors, we calculate the total energy of several different collinear magnetic configurations, and then project the $\mathrm{DFT}+U$ calculated total energies onto the below classical Heisenberg spin model,

$$
H=E_{0}+\sum_{\langle i, j\rangle} J_{i j}^{(1)} \boldsymbol{S}_{i, A} \cdot \boldsymbol{S}_{j, B}+\sum_{\langle\langle i, j\rangle\rangle} J_{i j}^{(2)} \boldsymbol{S}_{i, A} \cdot \boldsymbol{S}_{j, B} .
$$

Here, $\langle i, j\rangle$ indices refer to the nearest $A(\mathrm{Co})$ and $B(\mathrm{Cr})$ sublattices, whereas $\langle\langle i, j\rangle\rangle$ indices refer to the second-near neighbor $A$ and $B$ sublattices. $\boldsymbol{S}_{i, A}$ and $\boldsymbol{S}_{j, B}$ denote the spins at $A$ and $B$ magnetic sites. $E_{0}$ represents a constant energy term. A doubled unit cell was used to extract the second-neighbour magnetic exchange constants in bulk. However, for $n=1 \mathrm{CoCr}_{2} \mathrm{O}_{4} / \mathrm{MgAl}_{2} \mathrm{O}_{4}$ heterostructure, only three relevant nearest-neighbor magnetic exchange constants were considered.

Figure S8 shows the fitting of our model spin Hamiltonian with the DFT $+U$ calculated energies 

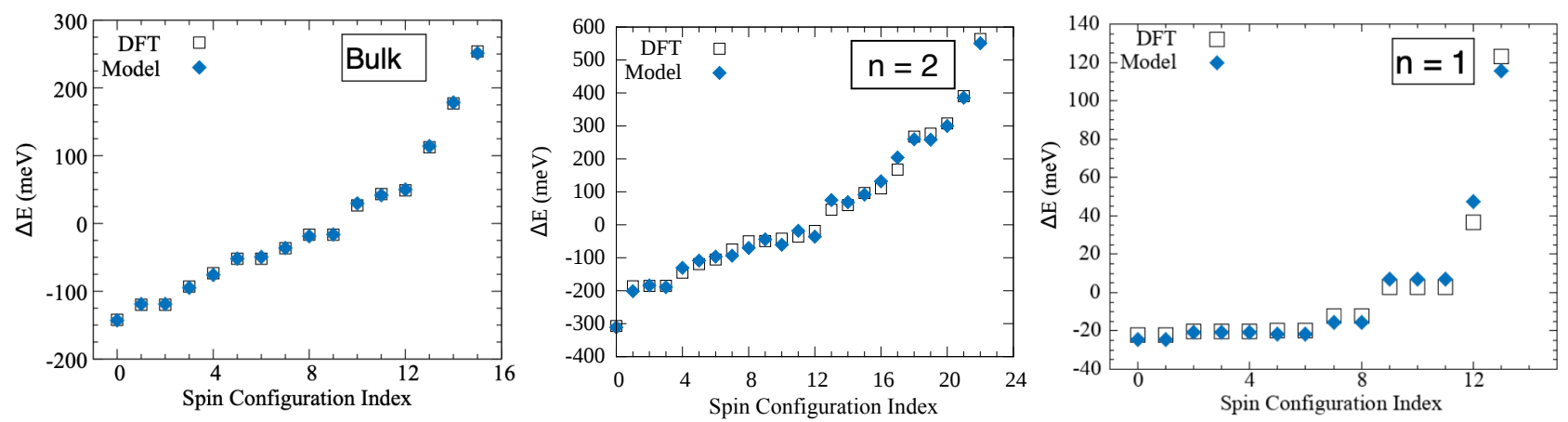

Figure S8: Fitting of the DFT $+U$ data with our classical Heisenberg spin model, given in Eq. 1, for bulk $\mathrm{CoCr}_{2} \mathrm{O}_{4}, n=2$, and $n=1$ slab configurations. Numerous competing magnetic ground state spin configurations can be noticed for $n=1$ case. We note that only three relevant nearestneighbor $J_{i j}^{(1)}$ parameters were used for fitting the $n=1$ data, whereas five $\left(J_{i j}^{(1)}\right.$ and $J_{i j}^{(2)}$ ) exchange coupling parameters were used to fit the bulk and $n=2$ data, as listed in Table S1.

for bulk, $n=2$, and $n=1$ cases. A reasonably good fitting was obtained for all three cases, which boosts our confidence in the prediction of the fitting parameters $J_{i j}$. Thus obtained values of magnetic exchange parameters for bulk, $n=2$, and $n=1$ cases are listed in Table $\mathrm{S} 1$. In our model, a positive $J_{i j}$ denotes antiferromagnetic (AFM) exchange interaction, whereas a negative $J_{i j}$ denotes ferromagnetic (FM) exchange interaction. Our predicted nearest neighbor exchange interactions $J_{i j}^{(1)}$ for bulk $\mathrm{CoCr}_{2} \mathrm{O}_{4}$ are in good agreement with the previous reports. ${ }^{10,11,13,14}$

For all three cases, the $J_{A B}^{(1)}$ is the most dominating exchange interaction (AFM) which is primarily responsible for establishing a Neél type AFM order in the studied systems. However, the subsequent $J_{B B}^{(1)}$ and $J_{A A}^{(1)}$ AFM terms introduce magnetic frustration at the $A$ and $B$ sites. We note that a larger ratio of $J_{B B}^{(1)} / J_{A B}^{(1)}$ implies the presence of strong magnetic frustrations, as suggested by the LKDM theory. ${ }^{15}$ Our analysis reveals that the $J_{B B}^{(1)} / J_{A B}^{(1)}$ ratio, which governs the magnetic frustration in this system, enhances upon the dimensional reduction $\left[J_{B B}^{(1)} / J_{A B}^{(1)}=0.43\right.$ (bulk), 0.52 $(n=2)$, and $0.98(n=1)$ ], indicating an anomalously large enhancement in magnetic frustration for $n=1$ case. Consequently, a distinct magnetic ground state is hard to stabilize in $n=1 \mathrm{uc}$ thick film even at ultralow temperatures. This is consistent with our experimental results as well as with our Monte-Carlo simulations results presented below.

Here, we must note that the DFT $+U$ computed total energies for bulk, $n=2$, and $n=1$ cases 
involve structures having different chemistry in regard to the total number of orbitals, total number of electrons, pseudopotentials, and interface-induced effects. Moreover, only three exchange coupling parameters [first neighbors: $J_{A A}^{(1)}, J_{A B}^{(1)}$, and $J_{B B}^{(1)}$ ] were used for fitting the $\mathrm{DFT}+U$ energy data for $n=1$ case, whereas five exchange coupling parameters [first and second neighbors: $J_{A A}^{(1)}, J_{A B}^{(1)}$, $J_{B B}^{(1)}, J_{A B}^{(2)}$, and $\left.J_{B B}^{(2)}\right]$ were used to fit the $\mathrm{DFT}+U$ data for the bulk and $n=2$ cases. Therefore, the absolute values of the extracted exchanges constants cannot be "assertively" compared on the same footing. However, their ratio for each systems can be compared since the ratio of the different exchange constants (e.g., $\left.J_{B B}^{(1)} / J_{A B}^{(1)}\right)$ rules out the differences arising due to the numerical details of the DFT $+U$ calculations for the studied systems.

Table S1: The first-neighbor $J_{i j}^{(1)}$, and the second-neighbor $J_{i j}^{(2)}$ magnetic exchange constants (in meV units) obtained using $\mathrm{PBE}+U$ for bulk $\mathrm{CoCr}_{2} \mathrm{O}_{4}, n=2$, and $n=1$ cases. Note that only three relevant first-neighbor $J_{i j}^{(1)}$ parameters were used for fitting the $n=1$ data.

\begin{tabular}{|c|c|c|c|}
\hline$J_{i j}^{(N)}$ & bulk & $n=2$ & $n=1$ \\
\hline$J_{A A}^{(1)}$ & 0.70 & 4.16 & 2.19 \\
$J_{A B}^{(1)}$ & 4.85 & 12.3 & 4.56 \\
$J_{B B}^{(1)}$ & 2.10 & 6.35 & 4.49 \\
$J_{A B}^{(2)}$ & -0.61 & -1.03 & - \\
$J_{B B}^{(2)}$ & -0.04 & -0.55 & - \\
$J_{B B}^{(1)} / J_{A B}^{(1)}$ & 0.43 & 0.52 & 0.98 \\
\hline
\end{tabular}

We note that $J_{A A}^{(1)}$ is the weakest first-neighbor exchange in bulk $\mathrm{CoCr}_{2} \mathrm{O}_{4}$ (corresponding CoCo bond length $=3.66 \AA$ ), therefore, we consider the second-neighbor $J_{A A}^{(2)}$ term to be negligible due to the large Co-Co bond length $(\sim 6.0 \AA)$ for the second-neighbour Co-Co interactions.

Notably, the second-neighbor exchange interaction terms, $J_{A B}^{(2)}$ and $J_{B B}^{(2)}$, are FM in nature, which tend to suppress the magnetic frustration arising due to the first-neighbor AFM $J_{i j}^{(1)}$. Thus, the presence of $J_{i j}^{(2)}$ terms in bulk counterbalances the magnetic frustration and facilitates a clear magnetic order (with canted spins due to the non-zero $J_{B B}^{(1)}$ and $J_{A A}^{(1)}$ terms) in the bulk. However, in the $n=1$ slab case, the number of second-neighbor exchange interaction terms along the outof-plane direction of film are truncated due to the presence of the nonmagnetic spacer layer. This scarcity of second-neighbor FM $J_{i j}^{(2)}$ terms, required to balance the magnetic frustration, yields 
strong uncompensated magnetic frustration in the $n=1$ slab. Due to this reason, a distinct magnetic ground state is hard to stabilize in $n=1$ film even at ultralow temperatures $(\sim 30 \mathrm{mK}$ in our case).

We further verify the aforementioned arguments by performing classical Monte-Carlo (MC) simulations for bulk and slab systems of $\mathrm{CoCr}_{2} \mathrm{O}_{4}$. Our Monte-Carlo simulations show that a distinct magnetic ground state can be achieved for bulk system at low temperatures, however, there exists plenty of competing magnetic ground states for $n=1$ and $n=2$ unit cell thick films of $\mathrm{CoCr}_{2} \mathrm{O}_{4}$, as discussed below.

\section{Monte-Carlo Simulations}

Finite temperature thermodynamics were obtained using a combination of parallel tempering moves ${ }^{17-20}$ and adaptive Metropolis steps. ${ }^{21,22}$ The combination of these two efficient updates led us to achieve thermalization even at very low-temperatures. Our Monte-Carlo simulations were performed using the layered cell (see figure S7 (b)), which has 2 Co spins $S_{A}$ and $4 \mathrm{Cr}$ spins $\boldsymbol{S}_{B}$, first on the bulk system, using periodic boundary conditions in all directions, with $N_{x}=N_{y}=N_{z}=N_{0}$ with $N_{0}$ of at most 16 cells, and then for the slab systems having periodic boundary conditions only along in-plane $x$ and $y$ directions.

We used a classical Heisenberg spin model with first and second nearest neighbors between A (Co atoms) and B (Cr atoms) sites, as given by the following spin Hamiltonian:

$$
\begin{aligned}
H & =J_{A A}^{(1)} \sum_{\langle i, j\rangle} \boldsymbol{S}_{i, A} \cdot \boldsymbol{S}_{j, A}+J_{B B}^{(1)} \sum_{\langle i, j\rangle} \boldsymbol{S}_{i, B} \cdot \boldsymbol{S}_{j, B} \\
& +J_{B B}^{(2)} \sum_{\langle\langle i, j\rangle\rangle} \boldsymbol{S}_{i, B} \cdot \boldsymbol{S}_{j, B} \\
& +J_{A B}^{(1)} \sum_{\langle i, j\rangle} \boldsymbol{S}_{i, A} \cdot \boldsymbol{S}_{j, B}+J_{A B}^{(2)} \sum_{\langle\langle i, j\rangle\rangle} \boldsymbol{S}_{i, A} \cdot \boldsymbol{S}_{j, B}
\end{aligned}
$$

with the magnetic exchange parameters predicted by the DFT calculations (Table S1) as an input 
for our Monte-Carlo simulations. Spins were considered classical since the size of the spins is $S=$ $3 / 2$, hence, quantum effects are not included in our model. However, we would like to mention that quantum effects could govern the underlying physics of this system at ultra-low temperatures. For all simulations, 30,000 MC updates per site were performed and discarded to achieve full thermalization. Another 30,000 steps were done to obtain thermodynamical observable such as energy, magnetization and specific heat. Error on obtained averages were calculated using the Jackknife method. ${ }^{17}$ At most, we ran 96 simulations in parallel at different temperatures, ranging from $T_{\text {low }}=0.001 J_{A B}^{1}$ to $T_{\text {low }}=3 J_{A B}^{1}$. For $J_{A B}^{1} \simeq 4.85 \mathrm{meV} \simeq 56 \mathrm{~K}$, this corresponds to a range from $56 \mathrm{mK}$ to $168 \mathrm{~K}$. Classical Monte-Carlo simulations at these low-temperatures are only provided in an exploratory manner, as it is quite possible that strong quantum effects become important before reaching such temperatures.

We find that the bulk system undergoes a single first-order phase transition at $T_{C} \simeq 85 \mathrm{~K}$. The low-temperature ordered state is a canted antiferromagnet, with $\boldsymbol{S}_{i, A} \cdot \boldsymbol{S}_{i, B}=\cos [\theta(T)]$. At $T=0$, the system goes to $\theta=\pi$, and the $\mathrm{Co}$ and $\mathrm{Cr}$ spins are anti-aligned in the unit cell such that $\boldsymbol{S}_{A}=-\boldsymbol{S}_{B}$, which is then repeated perfectly across the system. This result can be seen in the behavior of the total magnetization $M_{t o t}=\frac{1}{N}\left|\sum_{i} \sum_{j=1}^{6} \boldsymbol{S}_{i, j}\right|$ with $\boldsymbol{S}_{i, j}$ the $j$-th spin of the $i$-th unit cell (see Fig. S9). It is also evident in the canting measure behavior. The canting measure is defined as $C_{A B}=\frac{1}{N}\left|\sum_{i} \sum_{j_{A}=1}^{2} \sum_{j_{B}=3}^{6} \boldsymbol{S}_{i, j_{A}} \cdot \boldsymbol{S}_{i, j_{B}}\right|$ (see Fig. S9), and for a perfect AF state at zero temperature, will converge to 8 .

Simulations were also performed for two slab systems $(n=1,2)$, where $N_{x}=N_{y}=N_{0}$ with $N_{0}$ at most 30 cells, and slab thickness $N_{z}=1,2$. Periodic boundary conditions were maintained in the $x$ and $y$ directions, whereas an open boundary was kept in the $z$ direction. The unit cell was chosen in such a way that the $x, y, z$ axes are similar to the laboratory frame, and the orientation of layers corresponds to the ones obtained via thin-film deposition (see Fig. S7(b)). For both $n=1,2$ slabs, we find that the canted AFM bulk state at low temperature is avoided. Although the slabs go through a phase transition at a similar temperature as the bulk $T_{C}^{s l a b} \sim 50 \mathrm{~K}$, due to the activation of dominating first-neighbor $J_{A B}^{1}$ exchange interactions, there is a big distinction between the slab and 
bulk systems at low temperature. In the slab systems, a distinct magnetically ordered phase never really sets in, as evident in Figure S10, where significant energy and magnetization fluctuations are still present.

The following subsections present details on the Monte-Carlo algorithm used, and then specific measurements for the bulk and slab simulations, as well as a discussion of the thermalization.

\section{Monte-Carlo Algorithm:}

We used a combination of two algorithms. Firstly, we used a local Metropolis update, albeit modified a bit to achieve optimal sampling. ${ }^{21,22}$ In this method, one proceeds by randomly selecting a site $i$ and spin $\boldsymbol{S}_{i}$, and preparing a Gaussian flipped spin

$$
\boldsymbol{S}_{i}^{\prime}=\frac{\boldsymbol{S}_{i}+\boldsymbol{\Gamma}_{\sigma}}{\left|\boldsymbol{S}_{i}+\boldsymbol{\Gamma}_{\sigma}\right|}
$$

where, $\Gamma$ is a randomly generated vector according to a Gaussian distribution with variance $\sigma$. This leads to a new spin $\boldsymbol{S}_{i}^{\prime}$ that is within a solid angle of $2 \pi \sigma$ of the initial spin. This new spin then leads to a new energy for the system $E_{i}^{\prime}$ which is compared to the initial energy $E_{i}$. According to a usual Metropolis move, this is accepted with probability $p=\exp \left\{-\left(E_{i}^{\prime}-E_{i}\right) / T\right\}$. This move can be optimized at the beginning of the algorithm by calculating, for sites $A$ and $B$ respectively, and at all the temperatures sampled, the acceptance rate $R$ at which such moves lead to the new spin being accepted. Then, the variance $\sigma$ is modified using $\sigma^{\prime}=f \sigma$ with $f=\frac{0.5}{1-R}$. Applying this method a few times before the thermalization allows us to obtain variance $\sigma_{A}$ and $\sigma_{B}$ that are optimal since they lead to an acceptance ratio of $50 \%$. This usually has the effect of having very small variance at low temperature. A too large value of variance would lead to moves that depart too much from the original spin, leading to a lot of move rejections. This is optimal when most of the moves are efficient and accepted.

We ran our simulations for $M=96$ temperatures in parallel using the parallel tempering rou- 
tine. Temperatures $T_{i}$ were chosen using a geometric series $T_{i}=T_{1} R_{t}^{(i-1)}$ with $R_{t}=\left(\frac{T_{M}}{T_{1}}\right)^{1 /(M-1)}$ and $T_{1}=0.001 J_{A B}^{(1)}$ and $T_{M}=3 J_{A B}^{(1)}$. In a parallel tempering move, one considers whether changing the assignment of a temperature to a given configuration is preferred. Let us consider two systems $\left(\mathcal{S}_{i}, T_{i}, E_{i}\right)$ and $\left(\mathcal{S}_{j}, T_{j}, E_{j}\right)$ with $i, j$ being two neighboring temperatures in the temperature series. Both configurations $\mathcal{S}_{i}$ and $\mathcal{S}_{j}$ first undergo 100 Metropolis updates per site, yielding a final energy $E_{i}$ and $E_{j}$ corresponding to the configuration. One then considers exchanging $T_{i} \leftrightarrow T_{j}$ based on a probability $P_{i j}$. One first computes $\Delta_{i j}=\left[\left(E_{i}-E_{j}\right)\left(\frac{1}{T_{i}}-\frac{1}{T_{j}}\right)\right]$ and then the temperature switch is then accepted with probability $P_{i j}=\exp \left\{\Delta_{i j}\right\}$. If such a move is accepted, the spin configuration $\mathcal{S}_{i}$ is now run at temperature $T_{j}$, and vice versa.

The use of parallel tempering in a Monte-Carlo algorithm has been shown to greatly improve thermalization of the sample, as well as avoiding the formation of glassy phases. ${ }^{17-20}$ This is achieved because configurations reach a great level of ergodicity, having a lot of opportunity to explore the large phase space of the system.

For all simulations, 30,000 MC updates per site were performed and discarded to achieve full thermalization. Another 30,000 steps were done to obtain thermodynamic observables. Error on such obtained measurements were calculated using the Jackknife method. ${ }^{17}$ Measurements that were taken were the energy per site $E$, the specific heat per site $C_{v}$, the sublattice and total magnetizations $M_{A}, M_{B}$ and $M_{t o t}$ and its susceptinility $\chi_{t o t}$, as well as the canting measurement $C_{A B}$. Definitions of the measurement is given below, with $N$ understood as being the total number of supercells in the system. 


$$
\begin{aligned}
E & =\frac{H}{N} \\
C_{v} & =\frac{\langle E\rangle^{2}-\left\langle E^{2}\right\rangle}{N T^{2}} \\
M_{A} & =\frac{1}{N}\left|\sum_{i} \sum_{j_{A}=1}^{2} \boldsymbol{S}_{i, j_{A}}\right| \\
M_{B} & =\frac{1}{N}\left|\sum_{i} \sum_{j_{B}=1}^{4} \boldsymbol{S}_{i, j_{B}}\right| \\
M_{t o t} & =\frac{1}{N}\left|\sum_{i}\left(\sum_{j_{A}=1}^{2} \boldsymbol{S}_{i, j_{A}}+\sum_{j_{B}=1}^{4} \boldsymbol{S}_{i, j_{B}}\right)\right| \\
\chi_{t o t} & =\frac{\left\langle M_{t o t}\right\rangle^{2}-\left\langle M_{t o t}^{2}\right\rangle}{N T} \\
C_{A B} & =\frac{1}{N}\left|\sum_{i} \sum_{j_{A}=1}^{2} \sum_{j_{B}=1}^{4} \boldsymbol{S}_{i, j_{A}} \cdot \boldsymbol{S}_{i, j_{B}}\right|
\end{aligned}
$$

\section{Results for the Bulk System}

In this section we examine the results for our bulk simulations. These were performed on $N_{x}=$ $N_{y}=N_{z}=L$ supercells, with periodic boundary conditions in each direction. Each supercell consists of $2 A$ sites and $4 \mathrm{~B}$ sites. The results are provided in Fig. S9, where we show the specific heat, magnetization, susceptibility and canting behavior for over two decades of temperature. The temperature axis is obtained using the values for the $J$ interactions in kelvin $(\mathrm{K})$, and for the specific heat and the susceptibility, we use a logarithmic axis for the temperature to accentuate the low-temperature phase.

We see that there is a very sharp transition at $T_{C} \simeq 85 \mathrm{~K}$ due to the activation of $J_{A B}^{1}$, and we have $C_{v}^{\max } \propto L^{3}$ behaviour. Such a scaling behaviour is a strong indicator of a first order transition. Looking at the magnetization, it is clear that the low-temperature ordered state is a canted antiferromagnet, with $\boldsymbol{S}_{i, A} \cdot \boldsymbol{S}_{i, B}=\cos \theta(T)$. The low-temperature phase has a finite and continuously varying magnetization of the $A$ and $B$ sublattices as well as $M_{t o t}$, although the change 
(a)

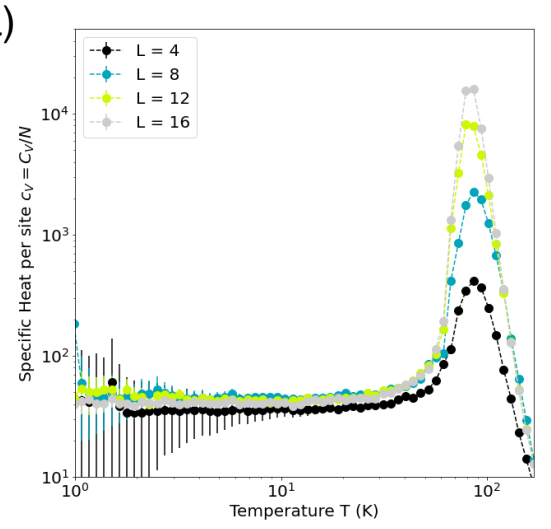

(b)

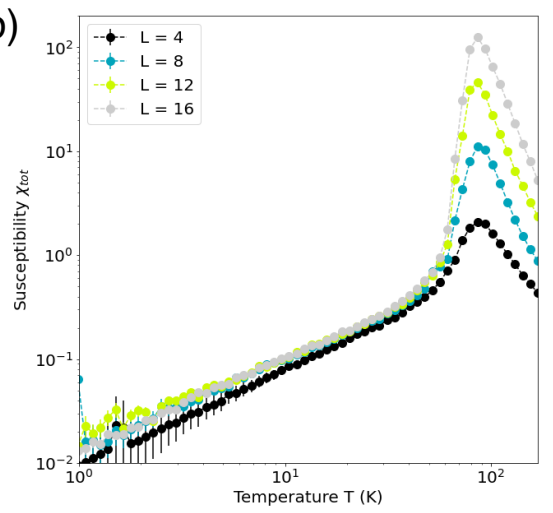

(c)

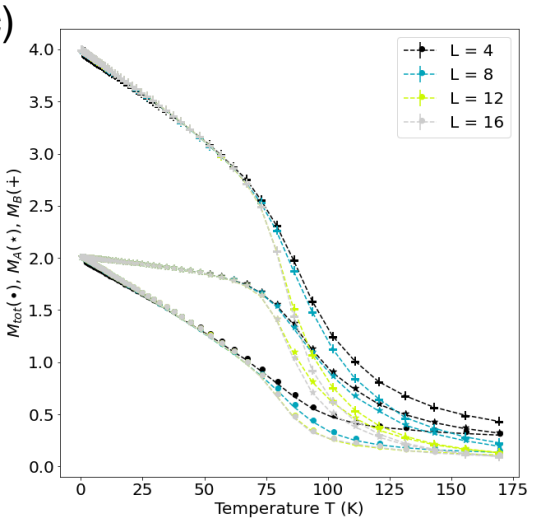

(d)

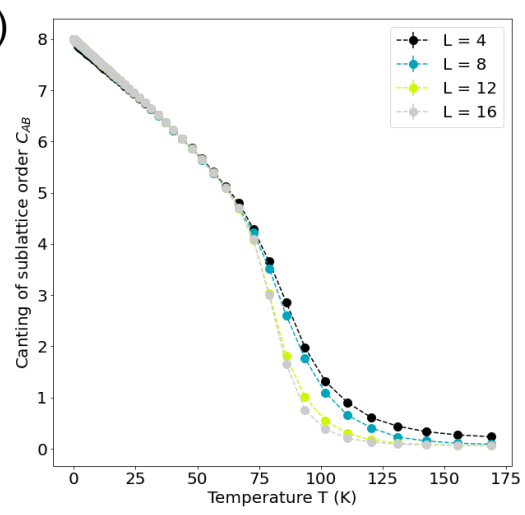

Figure S9: Thermodynamic results for the bulk simulations. Results for (a) specific heat per site, (b) susceptibility, (c) magnetization, and (d) canting angle measurements for bulk $\mathrm{CoCr}_{2} \mathrm{O}_{4}$ system. 
is mainly driven by the $B$ sites. The canted AF state can be clearly seen in the behavior of $C_{A B}$, which jumps from 0 to a finite value at $T_{C}$, and then progressively increases to reach a perfect AFM state at $T=0$. This canting measure, in a state where the $B$ sites are tilted $\theta$ degrees away from the $A$ sites, can be equal to $C_{A B} \simeq 8|\cos \theta|$, i.e. at $T=0$ we have a perfect AFM state. Finally, observation of the susceptibility of $M_{t o t}$ yields the same conclusions as the specific heat, and we are confident in the presence of a first-order temperature driven transition at $T_{C} \simeq 85 \mathrm{~K}$ to a canted AFM state in the bulk system, which is as expected from our DFT calculations.

\section{Results for the Slab Systems}

We now shift our focus to the slab simulation results. The format of the supercell used is such that stacking $2 \mathrm{D}$ layers in the $z$ direction replicates the laboratory samples created by deposition. In this section, we used samples with $L_{x}=L_{y}=L$, and $L_{z}=1,2$ for the two different slabs created (mono and double layer). Periodic boundary conditions were set in the $x$ and $y$ directions, and open boundary in the $z$. In the results presented below, we used $L=12,16,20$, and 30. MC runs on smaller systems were also performed but the obtained results were too sensitive to the boundary conditions. Firstly, we present the energy and the specific heat per site for such systems in Fig. S10. Whereas the bulk system's internal energy tends to -28.92 per site (the energy units are $J_{A B}^{(1)}$, the slab systems are unable to reach such an energy, which corresponds to a pure AFM state, the $T=0$ bulk ground state. What can be seen from the energy is that there is a broad feature associated with the bulk first-order transition at roughly the same critical temperature $\left(T_{C}^{s l a b} \sim 50 K\right)$. There is also a very sharp downturn at extremely low temperature, where the system is still trying to establish the $T=0$ ground state. However, as it can be seen in the specific heat plots, this low-temperature plot is permeated by a lot of energy fluctuations. Since $C_{V}=\sigma_{E} / T^{2}$, where $\sigma_{E}$ is the energy fluctuations, the specific heat plots (Fig. S10) reveal that there is simply a pervasive amount of energy fluctuations at low temperature preventing the settling of a distinct magnetic order, even in the presence of optimal MC algorithms. 
(a)

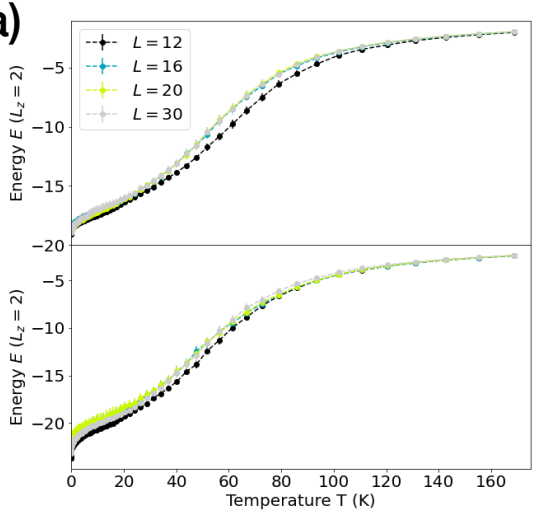

(c)

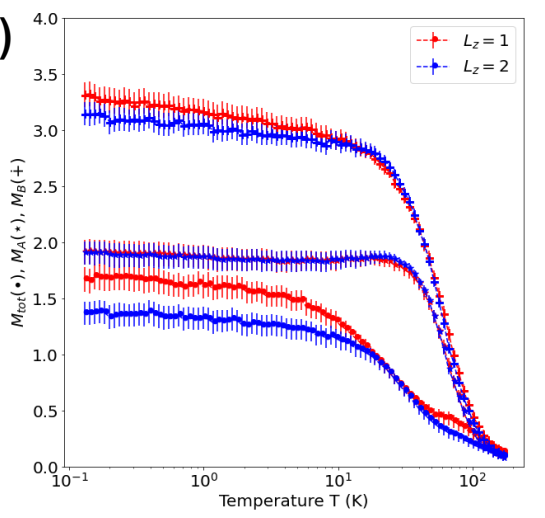

(e)

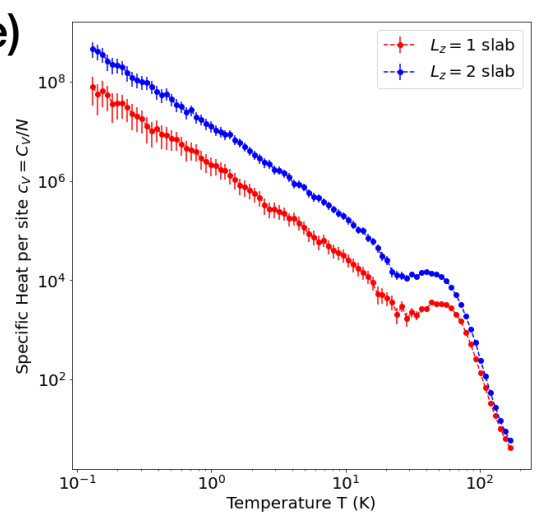

(b)

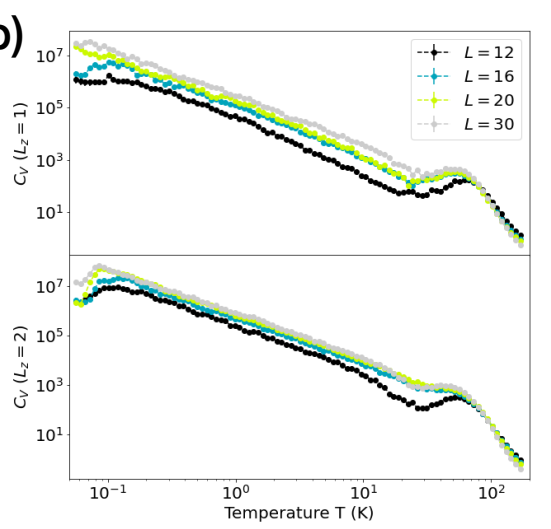

(d)

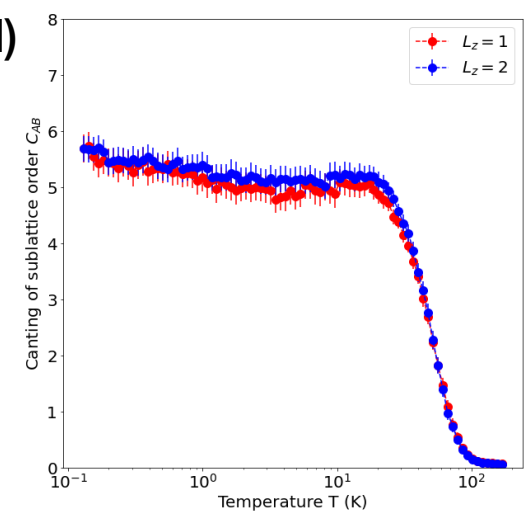

(f)

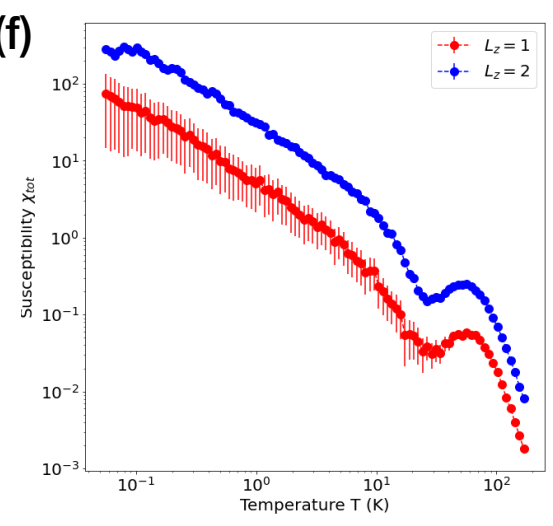

Figure S10: Thermodynamic results for the slab simulations. In the first row, results for different system sizes for $L_{z}=1$ (up) and $L_{z}=2$ (down), for (a) the energy per site, and (b) the specific heat. In the other figures, we show the data for $L_{x}=L_{y}=L=30$, while varying $L_{z}$. We have (c) the magnetizations (A (start), B (plus) sublattices and total (circles)), (d) the canting angle, (e) the specific heat per site, and (f) the total susceptibility data for slab systems. 
These results are also consistent with the magnetization and canting $C_{A B}$ versus temperature data for the slab systems, which are shown in Fig. S10. In this figure, we choose to show only the results for our simulations on $L_{x}=L_{y}=30$, our largest sample, for clarity. It is clear that although $M_{A}$ is able to reach its $T=0$ value where all $A$ spins are aligned, the $B$ spins cannot do the same and are plagued by large amount of fluctuations. This results in a finite canting state, but one that never really reaches the low-temperature bulk value. This is a clear indicator that the lower dimensionality samples are much more frustrated, and hence, the bulk magnetically ordered state cannot be realized.

An important question is whether we truly have achieved thermalization. In all our simulations, we performed 30,000 measurements, after each MC step, where every 100 steps we performed a parallel tempering move. In a true thermalized sample, the average value obtained in the first quarter of those measurements should not be drastically different from the one obtained in the last quarter. In Fig. S11 we show the energy per site and total magnetization for the two slab samples at $L=30$, for 4 blocks of our measurements (each is in different colors). It is clear that none of the bins show radical departure from the others and that the averages obtained above reflect the ones of a thermalized sample. The remain large error bars are due to the computational power at our disposal.
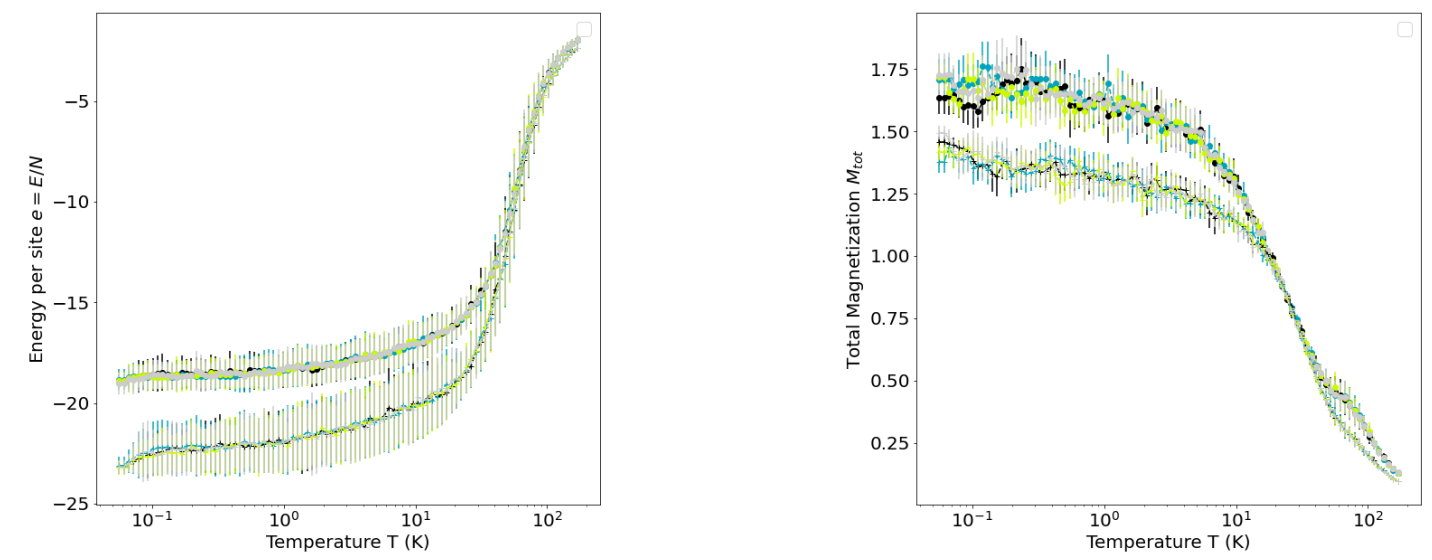

Figure S11: Measurements of the energy (left), and the total magnetization (right) for $L_{z}=1$ (circles) and $L_{z}=2$ (plus), for 4 different bins of the measurements. 


\section{References}

(1) X. Liu et al., Appl. Phys. Lett. 106, 071603 (2015).

(2) X. Liu, S. Middey, Y. Cao, M. Kareev, and J. Chakhalian, MRS commun. 6, 133-144 (2016).

(3) R. V. Chopdekar et al., J. Magn. Magn. Mater. 322, 2915-2921 (2010).

(4) G. Kresse and J. Furthmüller, Phys. Rev. B 54, 11169-11186 (1996).

(5) G. Kresse and J. Furthmüller, Comput. Mater. Sci. 6, 15-50 (1996).

(6) P. E. Blöchl, Phys. Rev. B 50, 17953-17979 (1994).

(7) G. Kresse and D. Joubert, Phys. Rev. B 59 1758-1775 (1999).

(8) J. P. Perdew, K. Burke, and M. Ernzerhof, Phys. Rev. Lett. 77, 3865-3868 (1996).

(9) S. L. Dudarev, G. A. Botton, S. Y. Savrasov, C. J. Humphreys, and A. P. Sutton, Phys. Rev. B 57, 1505-1509 (1998).

(10) I. Efthimiopoulos, Z. T. Y. Liu, S. V. Khare, P. Sarin, T. Lochbiler, V. Tsurkan, A. Loidl, D. Popov, and Y. Wang, Phys. Rev. B 92, 064108 (2015).

(11) C. Ederer and M. Komelj, Phys. Rev. B 76, 064409 (2007).

(12) L. Wang, T. Maxisch, and G. Ceder, Phys. Rev. B 73, 195107 (2006).

(13) G. Lawes, B. Melot, K. Page, C. Ederer, M. A. Hayward, Th. Proffen, and R. Seshadri, Phys. Rev. B 74, 024413 (2006).

(14) J. A. Heuver, A. Scaramucci, Y. Blickenstorfer, S. Matzen, N. A. Spaldin, C. Ederer, and B. Noheda, Phys. Rev. B 92, 214429 (2015).

(15) D. H. Lyons, T. A. Kaplan, K. Dwight, and N. Menyuk. Phys. Rev. 126, 540-555 (1962).

(16) X. Liu et al. Nano Lett. 19, 8381-8387 (2019). 
(17) Helmut G. Katzgraber, arXiv: 0905.1629.

(18) G. T. Barkema and M. E. J. Newman, arXiv: cond-mat/9703179.

(19) D. J. Earl and M. W. Deem, Phys. Chem. Chem. Phys. 7, 3910 (2005).

(20) N. Rathore, M. Chopra, and J. J. de Pablo. J. Chem. Phys. 122, 024111 (2005).

(21) W Keith Hastings, Biometrika 57, 97-109 (1970).

(22) J. D. Alzate-Cardona, D. Sabogal-Suárez, R. F. L. Evans, and E. Restrepo-Parra, J. Phys.: Condens. Matter 31, 095802 (2019). 